\title{
Alterations in necroptosis during ALDH2-mediated protection against high glucose-induced H9c2 cardiac cell injury
}

\author{
TINGTING FANG ${ }^{1,2}$, RUIPING CAO ${ }^{1,2}$, WENLIAN WANG $^{3}$, HONGWEI YE $^{1,2}$, \\ LIN SHEN $^{2}$, ZHENGHONG LI $^{1}$, JUNFENG HU $^{3}$ and QIN GAO $^{1,2}$ \\ ${ }^{1}$ Department of Physiology and ${ }^{2}$ Science Research Centre, Bengbu Medical College, Bengbu, Anhui 233030; \\ ${ }^{3}$ Department of Respiratory and Critical Care Medicine, The First Affiliated Hospital of Bengbu \\ Medical College, Bengbu, Anhui 233004, P.R. China
}

Received December 14, 2017; Accepted June 12, 2018

DOI: $10.3892 / \mathrm{mmr} .2018 .9269$

\begin{abstract}
The aim of the present study was to investigate whether necroptosis occurs in high glucose (HG)-induced $\mathrm{H} 9 \mathrm{c} 2$ cardiac cell injury and whether the activation of aldehyde dehydrogenase 2 (ALDH2) can inhibit necroptosis. H9c2 cardiac cells were treated with $35 \mathrm{mM}$ glucose to establish a HG-induced cell injury model. Alda-1 $(20 \mu \mathrm{M})$, a specific activator of ALDH2 and necrostatin-1 (Nec-1, $100 \mu \mathrm{M})$, an inhibitor of necroptosis were used to treat $\mathrm{H} 9 \mathrm{c} 2$ cardiac cells under HG conditions. Cell viability was measured using a Cell Counting Kit-8 assay and reactive oxygen species (ROS) generation was measured by the dihydroethidium staining method. ALDH2 activity was measured at $450 \mathrm{~nm}$. The mRNA and protein expression of ALDH2, necroptosis-associated genes, receptor-interacting protein (RIP)1, RIP3 and mixed lineage kinase domain like pseudokinase (MLKL), were analyzed by reverse transcription-quantitative polymerase chain reaction and western blotting. The expression of cleaved caspase- 3 protein was also examined by western blotting. The results demonstrated that under HG conditions, cell viability, ALDH2 activity, mRNA and protein expression were decreased. Furthermore, ROS generation, mRNA and protein expression of RIP1, RIP3, MLKL and the protein expression of cleaved caspase-3 were increased. Treatment with Alda-1 or Nec-1 attenuated HG-induced downregulation of ALDH2 activity, mRNA and protein expression. In addition, RIP1, RIP3, MLKL mRNA, and protein expression were downregulated.
\end{abstract}

Correspondence to: Dr Junfeng Hu, Department of Respiratory and Critical Care Medicine, The First Affiliated Hospital of Bengbu Medical College, 287 Changhuai Road, Bengbu, Anhui 233004, P.R. China

E-mail: bbmchjf@126.com

Dr Qin Gao, Department of Physiology, Bengbu Medical College, 2600 Donghai Avenue, Bengbu, Anhui 233030, P.R. China

E-mail: bbmcgq@126.com

Key words: H9c2 cardiac cells, high glucose, aldehyde dehydrogenase 2 , necroptosis
Furthermore, Alda-1 but not Nec-1 decreased cleaved caspase-3 protein expression. Collectively these data indicated that activation of ALDH2 protected $\mathrm{H} 9 \mathrm{c} 2$ cardiac cells against HG-induced injury, partly by inhibiting the occurrence of necroptosis.

\section{Introduction}

Diabetes mellitus is one of the most chronic and severe non-communicable diseases. Hyperglycemia-induced cardiac injury is one of the serious complications of diabetes, which can lead to myocardial oxidative stress, inflammation, calcium overload, cell death and ultimately myocardial failure.

There are different forms of cell death, including necrosis, apoptosis, autophagy, necroptosis and mitoptosis. Recently, necroptosis as a form of regulated necrosis has attracted widespread attention (1). Apoptosis is a caspase-dependent process, as is widely established. During apoptosis, caspase activation is mediated by extrinsic signals that activate cell surface receptors or intrinsic signals that induce cytochrome c release from mitochondria. Similar to apoptosis, necroptosis is a form of regulated cell death, though it is not mediated by caspases. Necroptosis has been reported in the pathogenesis of various diseases, including myocardial ischemia, brain ischemia and inflammation (2).

Necroptosis is mediated by the receptor-interacting protein kinase (RIP)1 and RIP3-dependent pathway. The association between high glucose (HG)-induced injury and necroptosis has been investigated by Liang et al (3), who reported that RIP3 and reactive oxygen species (ROS) both participate in high glucose-induced inflammation in H9c2 cardiac cells (4); however, the detailed mechanism was unclear. Since ROS overload and necroptosis both participate in HG-induced cardiac cell injury, an interesting research question is whether interventions can be selected to inhibit the release of ROS and/or inhibit necroptosis in order to mediate a protective effect on the cardiac cells.

It was widely reported that the activation of aldehyde dehydrogenase (ALDH)2 has a number of implications on the brain, heart, lung, liver injury and cancer $(5,6)$. The previous studies identified that the activation of ALDH2 can attenuate diabetes-induced oxidative stress and myocardial injury as well 
as inhibiting apoptosis (7-9). However, it is unclear whether the activation of $\mathrm{ALDH} 2$ represses necroptosis as a mechanism for protecting cardiac cells. In the present study, the alterations in necroptosis in HG-induced injury of $\mathrm{H} 9 \mathrm{c} 2$ cardiac cells were observed and the association between ALDH2 and necroptosis was investigated.

\section{Materials and methods}

Cell culture. The myoblast $\mathrm{H} 9 \mathrm{c} 2$ rat cardiac cell line from the heart was purchased from Shanghai GeneChem Co., Ltd., (Shanghai, China). The cells were cultured in Dulbecco's modified Eagle's medium (DMEM; HyClone; GE Healthcare Life Sciences Logan, UT, USA) supplemented with $10 \%$ fetal bovine serum (HyClone GE Healthcare Life Sciences) and $1 \%$ penicillin-streptomycin solution (Beyotime Institute of Biotechnology, Shanghai, China) at $37^{\circ} \mathrm{C}$ in a humidified $5 \%$ $\mathrm{CO}_{2}$ atmosphere. The cells were grown until 70-85\% confluence was reached for subsequent experiments.

Chemicals and antibodies. Alda-1, the specific activator of ALDH2, necrostatin-1 (Nec-1), the specific inhibitor of necroptosis and the dihydroethidium (DHE) fluorescent probe were obtained from Sigma-Aldrich (Merck KGaA, Darmstadt, Germany). The mitochondrial ALDH2 activity assay kit was obtained from Abcam (Cambridge, UK). The ALDH2 antibody was obtained from Santa Cruz Biotechnology, Inc., (Dallas, TX, USA). The RIP1 and RIP3 antibodies were obtained from Abcam. The mixed lineage kinase domain like pseudokinase (MLKL) antibody was obtained from Affinity Biosciences (Cambridge, UK). The cleaved caspase-3 antibody was obtained from Cell Signaling Technology, Inc., (Danvers, MA, USA). The GAPDH antibody was acquired from Boster Biological Technology Co., Ltd. (Wuhan, China). A Cell Counting Kit-8 (CCK-8) assay kit was from Bestbio Life Technology (Shanghai, China; http://www.bestbio. com.cn; cat. no. BB-4202-1). Primers for ALDH2, RIP1, RIP3, MLKL and GAPDH were acquired from Sangon Biotech Co., Ltd. (Shanghai, China; sequences are presented in Table I). A RevertAid RT Reverse Transcription kit was purchased from Thermo Fisher Scientific, Inc., (Waltham, MA, USA). The SYBR ${ }^{\circledR}$ Premix DimerEraser ${ }^{\mathrm{TM}}$ (Perfect Real Time) was acquired from Takara Bio, Inc. (Otsu, Japan).

Experimental protocols. The cells were incubated at $37^{\circ} \mathrm{C}$ in a humidified $5 \% \mathrm{CO}_{2}$ atmosphere, the experiments were divided into 5 groups as follows: i) Normal control group, where H9c2 cardiac cells were cultured in DMEM supplemented with $10 \%$ fetal bovine serum; ii) a hypertonic control group (HPG), where $\mathrm{H} 9 \mathrm{c} 2$ cardiac cells were subjected to $35 \mathrm{mM}$ mannitol as an osmotic control for $24 \mathrm{~h}$; iii) a high glucose group (HG), where $\mathrm{H} 9 \mathrm{c} 2$ cardiac cells were subjected to $35 \mathrm{mM}$ glucose stress for $24 \mathrm{~h}$ to induce cell injury; iv) an HG+Alda-1 group where $\mathrm{H} 9 \mathrm{c} 2$ cardiac cells, which were subjected to HG stress were treated with $20 \mu \mathrm{M}$ Alda-1 (a specific activator of ALDH2) (9) for $24 \mathrm{~h}$ and $\mathrm{v}$ ) an $\mathrm{HG}+\mathrm{Nec}-1$ group, where $\mathrm{H} 9 \mathrm{c} 2$ cardiac cells that were subjected to HG stress were treated with $100 \mu \mathrm{M}$ Nec-1 for $24 \mathrm{~h}$.

The hypertonic control group was used to exclude the role of the hypertonic effect. The aim of the HG+Alda-1 group was to investigate whether the activation of ALDH2 can attenuate HG induced-cardiac cell injury. The purpose for the $\mathrm{HG}+\mathrm{Nec}-1$ group was to observe whether inhibiting necroptosis can attenuate HG-induced cardiac cell injury.

CCK- 8 assay. The CCK- 8 assay kit was used to detect cell viability, according to the manufacturer's protocol. In brief, H9c2 cardiac cells (100 $\mu \mathrm{l} /$ well) were seeded into 96-well plates at a density of $8 \times 10^{3}$ cells/plate and incubated at $37^{\circ} \mathrm{C}$ and $5 \%$ $\mathrm{CO}_{2}$ overnight. A total of $10 \mu \mathrm{lCC}-8$ solution was added to each well following the different treatments described above. Following 1-4 h incubation in the dark at $37^{\circ} \mathrm{C}$, cell viability was determined by measuring the absorbance at a wavelength of $450 \mathrm{~nm}$ using a microplate reader (BioTek Instruments, Inc., Winooski, VT, USA).

Detection of alterations in superoxide production by DHE staining. Superoxide production was evaluated using the DHE staining method. H9c2 cardiac cells were seeded in 6 -well plates at a density of $2 \times 10^{5}$ cells/plate. Following treatment with different interventions, the cells were incubated with $10 \mu \mathrm{M}$ DHE solution at $37^{\circ} \mathrm{C}$ for $30 \mathrm{~min}$ in a chamber in the dark at $37^{\circ} \mathrm{C}$ and then washed with PBS 2-3 times. The cells were subsequently incubated with $1 \mu \mathrm{g} / \mathrm{ml}$ DAPI at $37^{\circ} \mathrm{C}$ for $15 \mathrm{~min}$ in the dark. The fluorescent image and intensity of DHE was measured at $485 \mathrm{~nm}$ (excitation wavelength) and $590 \mathrm{~nm}$ (emission wavelength). DAPI was measured at $358 \mathrm{~nm}$ (excitation wavelength) and $461 \mathrm{~nm}$ (emission wavelength) with a fluorescent microscope camera (Olympus IX71; Olympus Corporation, Tokyo, Japan). Superoxide production was expressed as a mean fluorescence ratio (fluorescence of exposed cells/fluorescence of control cells) and the mean fluorescence intensity was analyzed with Image J software (version 1.48, National Institutes of Health, Bethesda, MD, USA).

Analysis of ALDH2 activity. ALDH2 activity may be determined by the Mitochondrial Aldehyde Dehydrogenase (ALDH2) Activity Assay kit (cat. no. ab115348; Abcam) and following the production of NADH in the following ALDH2-catalyzed reaction: Acetaldehyde $+\mathrm{NAD}^{+} \rightarrow$ acid + $\mathrm{NADH}$. The reaction reduces a colorless probe to a colored product with strong absorbance at $450 \mathrm{~nm}$. This experiment was conducted according to the manufacturer's protocol.

Detection of ALDH2, RIP1, RIP3 and MLKL levels by reverse transcription-quantitative polymerase chain reaction $(R T-q P C R)$. Total RNA in H9c2 cardiac cells was isolated using TRIzol ${ }^{\circledR}$ reagent following the manufacturer's protocol (Invitrogen; Thermo Fisher Scientific Inc.). The purity and concentration of total RNA were detected using a microplate reader (Biotek ${ }^{\mathrm{TM}}$ Epoch $^{\mathrm{TM}}$; Biotek Instruments, Inc.). A total of $3 \mu \mathrm{g}$ total RNA was used to synthesize cDNA according to the protocol of the RT-qPCR kit (cat. no. K1691; Thermo Fisher Scientific Inc.). The total volume of each tube was $20 \mu \mathrm{l}$. The mixture was placed on a PCR machine under conditions of $42^{\circ} \mathrm{C} 60 \mathrm{~min}$ and $70^{\circ} \mathrm{C}, 5 \mathrm{~min}$ to obtain the cDNA. The volume of reagents used was determined according to the protocol of the qPCR kit (cat. no. RR091A; Takara Bio Inc.): $2 \mu \mathrm{l}$ cDNA template $(50 \mathrm{ng} / \mu \mathrm{l}) ; 0.6 \mu \mathrm{l}$ upstream and downstream primers 
Table I. Primer sequences for reverse transcription-quantitative polymerase chain reaction.

\begin{tabular}{|c|c|c|c|}
\hline Gene & Primer & Sequence $\left(5^{\prime}-3^{\prime}\right)$ & Product size (bp) \\
\hline \multirow[t]{2}{*}{ ALDH2 } & Forward & GTGTTCGGAGACGTCAAAGA & 187 \\
\hline & Reverse & GCAGAGCTTGGGACAGGTAA & \\
\hline \multirow[t]{2}{*}{ RIP1 } & Forward & AGGTACAGGAGTTTGGTATGGGC & 123 \\
\hline & Reverse & GGTGGTGCCAAGGAGATGTATG & \\
\hline \multirow[t]{2}{*}{ RIP3 } & Forward & TAGTTTATGAAATGCTGGACCGC & 145 \\
\hline & Reverse & GCCAAGGTGTCAGATGATGTCC & \\
\hline \multirow[t]{2}{*}{ MLKL } & Forward & GCCACTGGAAAGATCCCGTT & 108 \\
\hline & Reverse & CAACAACTCGGGGCAATCCT & \\
\hline \multirow[t]{2}{*}{ GAPDH } & Forward & ACAGCAACAGGGTGGTGGAC & 255 \\
\hline & Reverse & TTTGAGGGTGCAGCGAACTT & \\
\hline
\end{tabular}

MLKL, mixed lineage kinase domain like pseudokinase; RIP, receptor-interacting protein kinase 1; ROS, reactive oxygen species; ALDH2, aldehyde dehydrogenase 2 .

(final concentration, $0.3 \mu \mathrm{mol} / \mathrm{l}$ ); $0.4 \mu \mathrm{l}$ Rox reference dye; and $10 \mu \mathrm{l}$ SYBR Premix DimerErase with the addition of enzyme-free water to a total volume of $20 \mu \mathrm{l}$. The thermocycling conditions were as follows: i) Pre-denaturation at $95^{\circ} \mathrm{C}$ for $30 \mathrm{sec}$; ii) denaturation at $95^{\circ} \mathrm{C}$ for $5 \mathrm{sec}$; iii) annealing at $60^{\circ} \mathrm{C}$ for $30 \mathrm{sec}$ and iv) extension at $72^{\circ} \mathrm{C}$ for $34 \mathrm{sec}$. The steps 2-4 were repeated for 40 cycles. The dissolution curve was automatically generated by the thermocycler. The internal control GAPDH was used for correction and the normal group was used as a control. The $2^{-\Delta \Delta C q}$ value was calculated as the relative gene expression level of each sample and the data was analyzed (10). The sequences of the primers used are presented in Table I and the reference gene was GAPDH.

Detection of ALDH2, RIP1, RIP3, MLKL and cleaved caspase-3 levels by western blotting. The expression of ALDH2, RIP1, RIP3, MLKL and cleaved caspase-3 proteins was detected by western blotting. H9c2 cardiac cells in each group were homogenized in the mixture buffer of radioimmunoprecipitation assay lysate (cat. no. P0013B; Beyotime Institute of Biotechnology) and PMSF $(0.1 \mathrm{mM})$ for $60 \mathrm{~min}$ on ice. The mixture was centrifuged at $1,0621 \mathrm{x} \mathrm{g}$ for $15 \mathrm{~min}$ at $4^{\circ} \mathrm{C}$ to obtain the total protein, and the concentration of protein was measured by bicinchoninic acid assay (cat. no. P0010; Beyotime Institute Biotechnology). Equal quantities of protein lysates $(40 \mu \mathrm{g})$ were electrophoresed on $10 \%$ SDS-PAGE and transferred to a polyvinylidene difluoride membrane. Following blocking by 5\% nonfat milk for $150 \mathrm{~min}$ at room temperature, the membrane was probed overnight at $4^{\circ} \mathrm{C}$ with primary antibodies against ALDH2 (1:500; cat. no. sc-100496), RIP1 (1:500; cat. no. ab106393), RIP3 (1:400; cat. no. ab62344), MLKL (1:500; cat. no. DF7412) and cleaved caspase-3 (1:300; cat. no. 9664). Following washing, the membrane was probed with the corresponding secondary antibodies (goat anti-rabbit IgG; 1:8,000; cat. no. AP132P; Merck KGaA) at the water of $37^{\circ} \mathrm{C}$ for $40 \mathrm{~min}$ then agitating (100 rpm for $20 \mathrm{~min}$ ) at room temperature. Finally, the membrane was visualized using the enhanced chemiluminescence method (Immobilon Western

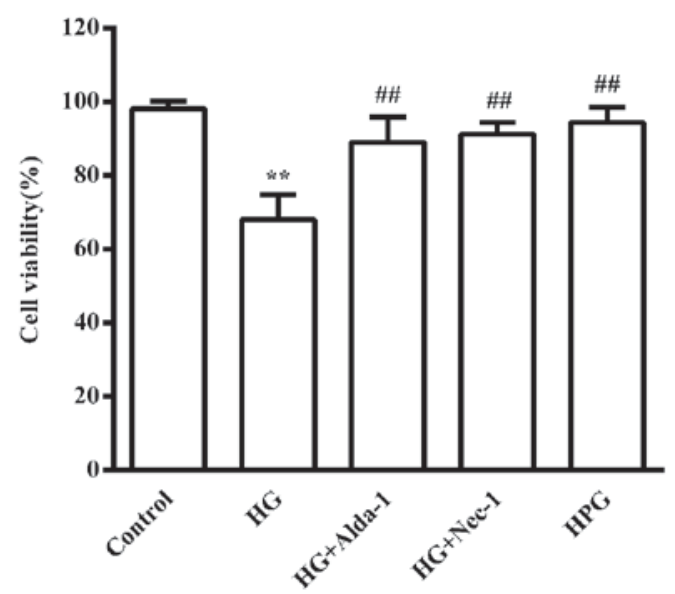

Figure 1. Alterations in the viability of $\mathrm{H} 9 \mathrm{c} 2$ cardiac cells determined by Cell Counting Kit- 8 assay in different groups. ${ }^{* *} \mathrm{P}<0.01$ vs. the control group; ${ }^{\# \#} \mathrm{P}<0.01$ vs. the $\mathrm{HG}$ group. Data are expressed as the mean \pm standard error of the mean $(n=6)$. Con, control; HG, high glucose; Nec-1, necrostatin-1; HPG, hypertonic control group.

Chemiluminescent HRP Substrate; cat. no. WBKLS0100; Merck KGaA). GAPDH (1:2,000; cat. no. PB0141) was used as a loading control. The autoradiographs were scanned using the ChemiDoc XRS Gel Image system and analyzed with the Image Lab software (version 3.0; Bio-Rad Laboratories, Inc., Hercules, CA, USA). The density value of each band was expressed as arbitrary units.

Statistical analysis. The data are presented as the mean \pm the standard error of the mean. Software used for statistical analysis was Graphpad Prism ${ }^{\circledR}$ (v6.0 for Windows; GraphPad Software, Inc., La Jolla, CA, USA). Statistical significance was determined using one-way analysis of variance followed by Newman-Keuls for comparisons between multiple samples. Experiments were repeated independently more than three times. $\mathrm{P}<0.05$ was considered to indicate a statistically significant difference. 

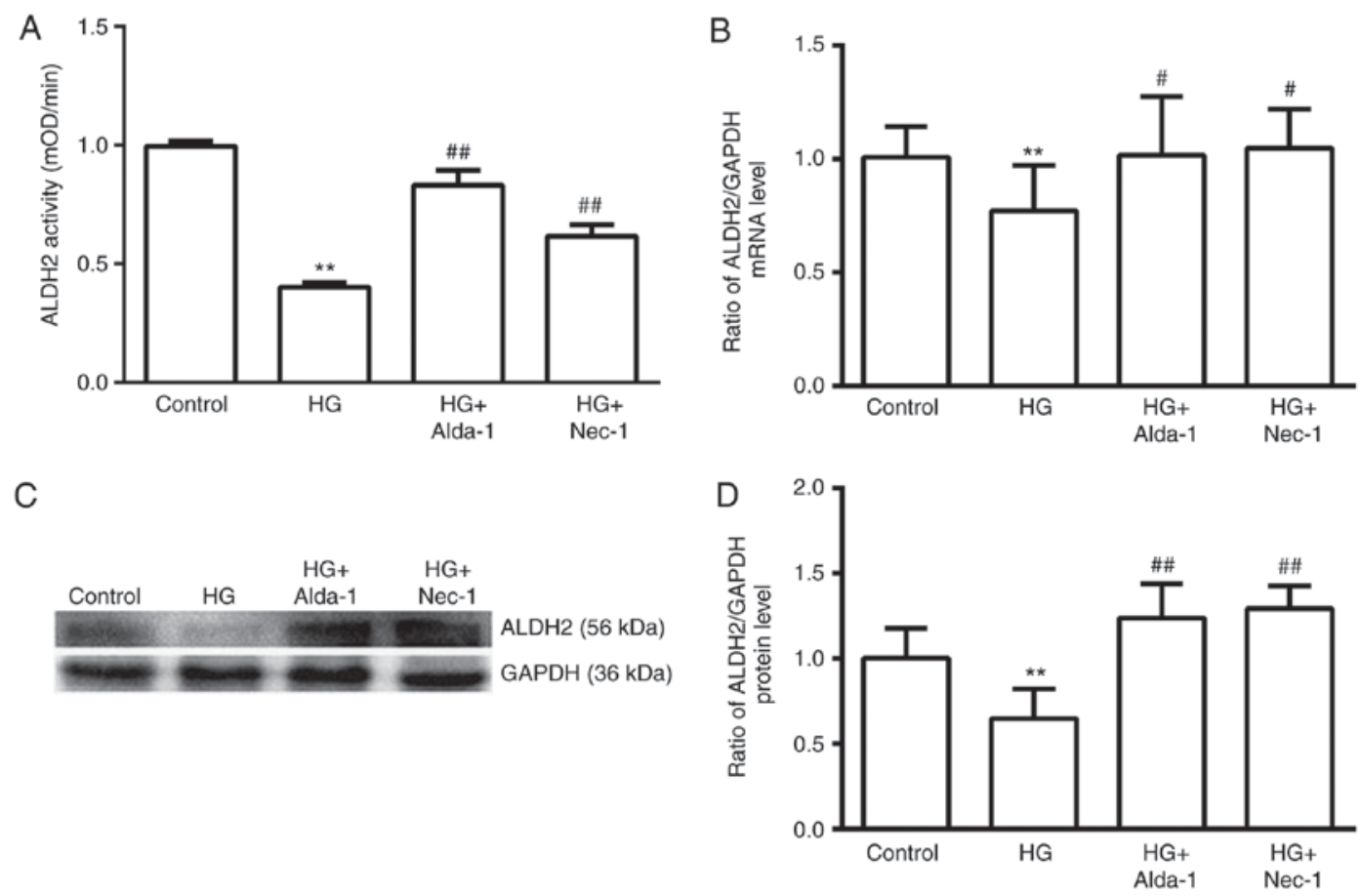

Figure 2. Alterations in ALDH2 activity and the expression of ALDH2 mRNA and protein in H9c2 cardiac cells in different groups. Statistical results for (A) ALDH2 activity and (B) mRNA expression. (C) Representative western blot bands and (D) statistical analysis of ALDH2 expression. ${ }^{* *} \mathrm{P}<0.01$ vs. the control group; ${ }^{~} \mathrm{P}<0.05,{ }^{\sharp \#} \mathrm{P}<0.01$ vs. $\mathrm{HG}$ group. Data are expressed as the mean \pm standard error of the mean $(\mathrm{n}=6)$. HG, high glucose; Nec-1, necrostatin-1; HPG, hypertonic control group; ALDH2, aldehyde dehydrogenase 2; OD, optical density.

\section{Results}

Alterations in cell viability in the different treatment groups. Compared with the control group, HG significantly decreased the viability of $\mathrm{H} 9 \mathrm{c} 2$ cardiac cells $(\mathrm{P}<0.01)$. Treatments with the ALDH2 activator, Alda-1 and the RIP1 inhibitor, Nec-1, significantly increased cell viability compared with the HG group $(\mathrm{P}<0.01)$. There was no marked difference in cell viability between the control and the HPG group (Fig. 1). As the hypertonic solution had no obvious effect on cell viability based on the CCK-8 results, further experiments were not performed on the HPG group.

Alterations in the activity, $m R N A$ and protein expression of $A L D H 2$ in different groups. Compared with the control group, HG intervention significantly decreased ALDH2 activity and ALDH2 expression at the mRNA and protein levels $(\mathrm{P}<0.01$; Fig. 2). In the Alda-1+HG and Nec-1+HG groups, the activity and mRNA and protein expression of ALDH2 were significantly increased compared with in the HG group $(\mathrm{P}<0.05$; Fig. 2). These results suggested that inhibiting necroptosis with Nec-1 induced increases in ALDH2 activity and expression, which was similar to the apparent mechanism of ALDH2 activation by Alda-1.

Alterations in ROS production in different treatment groups. Compared with the control group, DHE fluorescence intensity was significantly increased in the HG group, indicating an increase in $\operatorname{ROS}(\mathrm{P}<0.01)$. DHE fluorescence intensity was significantly decreased when the cells were treated with Alda-1 and Nec-1 under HG conditions ( $\mathrm{P}<0.01$; Fig. 3), which suggested that the activation of ALDH2 or the inhibition of necroptosis could reduce the over-production of ROS in HG-induced cardiac cell injury.

Alterations in RIP1, RIP3 and MLKL $m R N A$ and protein expression in different treatment groups. Compared with the control group, the expression of RIP1, RIP3 and MLKL at the mRNA and protein levels was significantly increased when the cells were subjected to HG intervention $(\mathrm{P}<0.05)$. In the Alda-1+HG and Nec-1+HG groups, the levels of RIP1, RIP3 and MLKL mRNA and protein expression were significantly decreased compared with in the HG group $(\mathrm{P}<0.05$; Figs. 4 and 5). The results suggested that necroptosis occurs in HG-induced cardiac cell injury. Furthermore, the activation of ALDH2 could downregulate necroptosis, which was similar to the apparent mechanism of inhibiting necroptosis via Nec-1.

Alterations in the expression of cleaved caspase-3 protein in different treatment groups. Compared with the control group, the expression of cleaved caspase-3 protein was increased in the HG group. Compared with the HG group, the expression of cleaved-caspase 3 protein was significantly decreased in the Alda-1+HG group $(\mathrm{P}<0.05)$. However, there was no difference between the HG group and the Nec-1+HG group (Fig. 5). The results suggested that the activation of ALDH2 could attenuate both the occurrence of apoptosis and necroptosis, while the inhibition of necroptosis by Nec-1 only attenuated necroptosis without having an effect on apoptosis (Fig. 6).

\section{Discussion}

In the present study, it was first observed that when H9c2 cardiac cells were subjected to HG stress, the activity and 
A

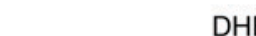

GG
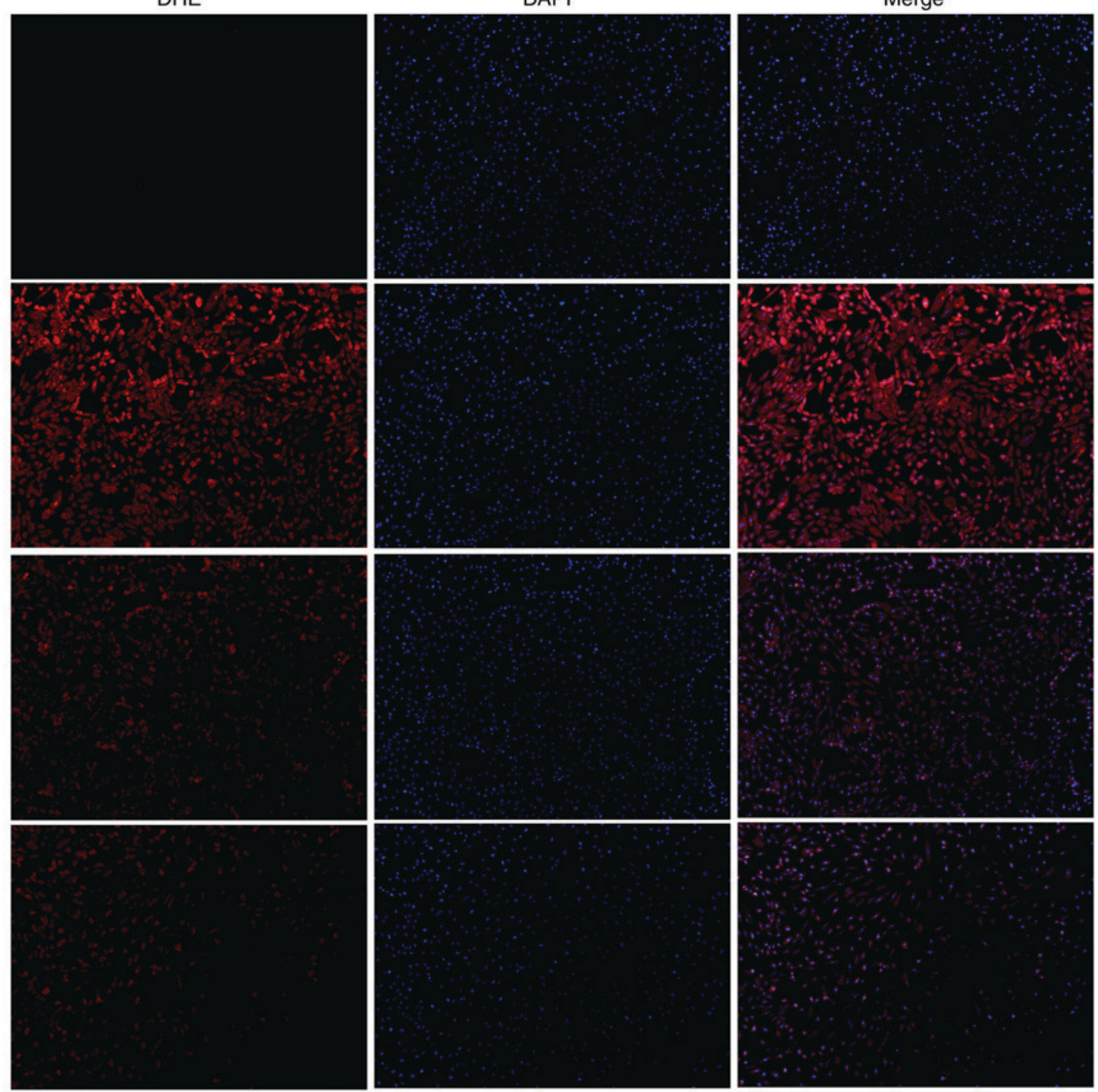

HG+Alda-1

$\mathrm{HG}+\mathrm{Nec}-1$
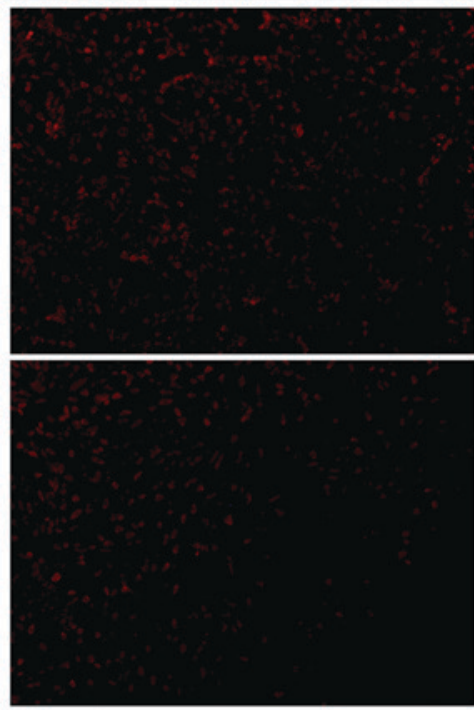

\section{B}

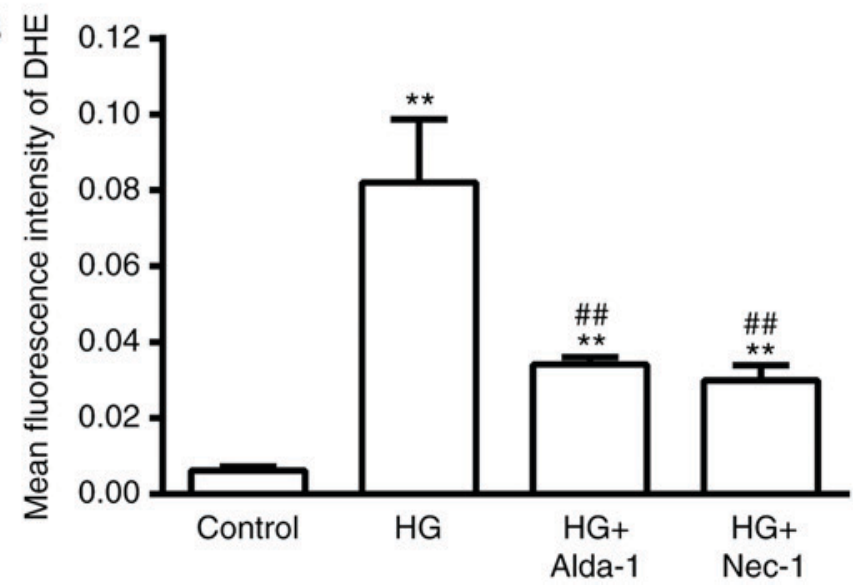

Figure 3. Alterations in superoxide production in H9c2 cardiac cells in different treatment groups. Representative stating with DHE and DAPI, and merged images (magnification x100). (A) Representative fluorescent images for the different treatment groups; (B) Statistical results of the mean DHE fluorescent intensity. ${ }^{* *} \mathrm{P}<0.01$ vs. the control group; ${ }^{\# \#} \mathrm{P}<0.01$ vs. the HG group. Data are expressed as the mean \pm standard error of the mean ( $=6$ ). DHE, dihydroethidium; HG, high glucose; Nec-1, necrostatin-1.

expression of ALDH2 were downregulated, which was accompanied by increases in the expression of key mediators of necroptosis (RIP1, RIP3 and MLKL). This suggested that hyperglycemia induced the occurrence of necroptosis in a model of HG-induced H9c2 cardiac cell injury. Then, it was observed that the necroptosis inhibitor, Nec-1 and the ALDH2 activator, Alda-1, increased the activity and expression of ALDH2 and inhibited necroptosis. This result suggested that 

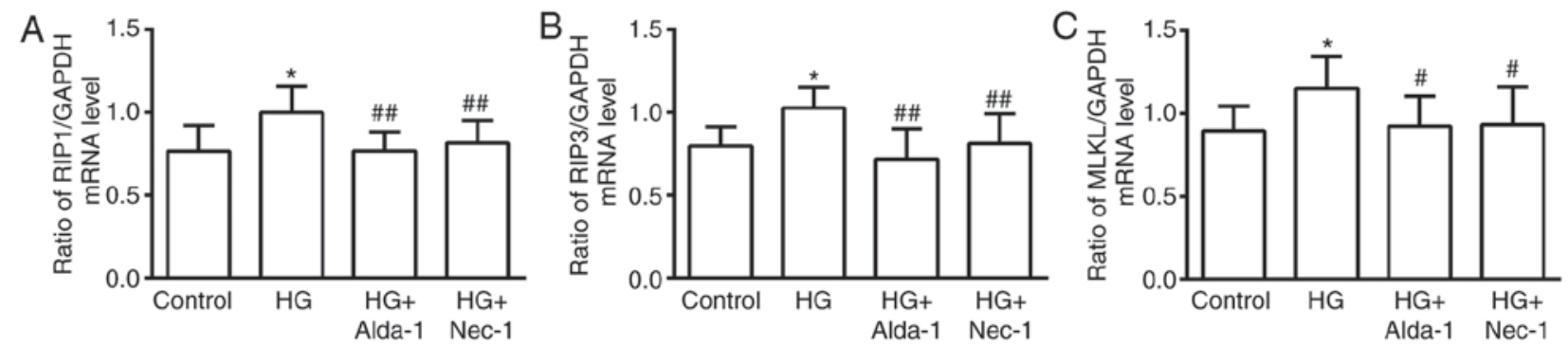

Figure 4. Expression of necroptosis genes. mRNA expression of (A) RIP1, (B) RIP3 and (C) MLKL in H9c2 cardiac cells in different groups. *P<0.05 vs. the control group; ${ }^{\#} \mathrm{P}<0.05,{ }^{\# \#} \mathrm{P}<0.01$ vs. the HG group. Data are expressed as the mean \pm standard error of the mean ( $\left.\mathrm{n}=6\right)$. HG, high glucose; MLKL, mixed lineage kinase domain like pseudokinase; RIP, receptor-interacting protein kinase.

A

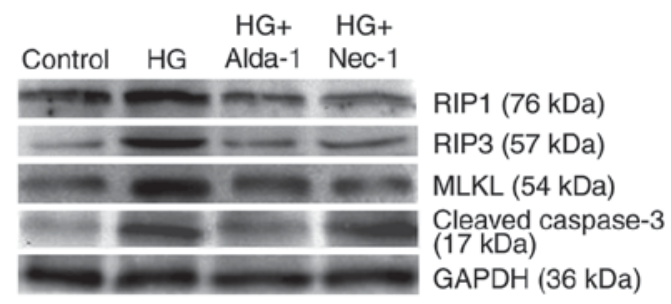

B

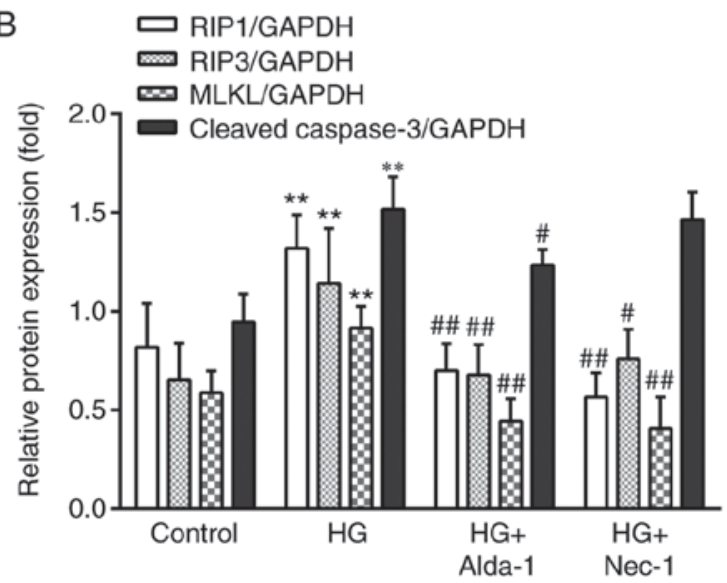

Figure 5. Alterations in the expression of RIP1, RIP3, MLKL and cleaved caspase-3 proteins in $\mathrm{H} 9 \mathrm{c} 2$ cardiac cells in different groups. (A) Representative western blots of RIP1, RIP3, MLKL and cleaved caspase-3 proteins. (B) Statistical analysis of the optical density values. ${ }^{* *} \mathrm{P}<0.01$ vs. the control group; ${ }^{\#} \mathrm{P}<0.05,{ }^{\# \#} \mathrm{P}<0.01$ vs. the HG group. Data are expressed as the mean \pm standard error of the mean $(n=6)$. HG, high glucose; MLKL, mixed lineage kinase domain like pseudokinase; RIP, receptor-interacting protein kinase 1; Nec-1, necrostatin-1.

the activation of ALDH 2 could protect cardiac cells by inhibiting RIP1/RIP3/MLKL-mediated necroptosis.

Recently, it has been reported that necroptosis (also named programmed necrosis) is one form of regulated cell death, which occurs in a number of diseases. Necroptosis is mediated by RIP1, RIP3 and MLKL, which forms the RIP1-RIP3-MLKL complex termed, the 'necrosome'. This complex is considered an important mediator of the necroptotic pathway (11-13).

ROS are small and highly reactive molecules with important signaling transduction functions when maintained at proper cellular concentrations. ROS levels are increased when the body is in a pathological state and the accumulation of ROS can elicit necroptosis as well as apoptosis (14-16). ROS production is considered as an effector of necroptosis in primary red blood cells, Jurkat T cells and U937 monocytes when they are subjected to HG stress (17). ROS may also be considered as an effector of necroptosis (18). ALDH2 is the mitochondrial isoform of the ALDH superfamily and is located in the mitochondrial matrix for removal of aldehyde substrates (19). The cardioprotective role of ALDH2 has been noted in epidemiological and experimental studies, ALDH2 mutation reduces ALDH2 activity and is associated with cardiovascular, neurological, and digestive complications $(19,20)$. The activation of ALDH2 can attenuate diabetes-induced myocardial injury $(7,8,21)$. ALDH2 had been widely recognized as a protective factor during organ injury triggered by different causes and one of its important protective mechanisms is inhibition of the overproduction of ROS (4). However, there are a limited number of reports regarding ALDH2 and necroptosis. Therefore, the present study aimed to observe the alterations in ROS, ALDH2 and necroptosis, and to analyze the likely mechanisms that are involved in HG-induced cardiac cell injury using H9c2 cells.

In the present study, it was observed that when $\mathrm{H} 9 \mathrm{c} 2$ cardiac cells were subjected to HG stress, which was accompanied by a decrease in cell viability and ROS production, ALDH2 activity was decreased and the levels of ALDH2 mRNA and protein expression were downregulated. Additionally, the levels of RIP1, RIP3 and MLKL mRNA and protein expression were all increased. These results suggested that hyperglycemia induced necroptosis in H9c2 cardiac cells, which was accompanied by a decline in ALDH2 activity and expression, and an increase in ROS production. These results suggest that necroptosis and ALDH2 may be linked.

The inhibition of necroptosis can exert beneficial effects both in vivo and ex vivo. Nec-1 is a specific inhibitor of necroptosis that inhibits the interaction between RIP1 and RIP3, Nec-1 can mediate the protective effects in myocardial and brain ischemic injury in adult rodent models (22-25). Cardiac cells under HG stress were treated with Nec-1 in order to verify that necroptosis occurred under hyperglycemic conditions and to determine whether inhibiting necroptosis can protect cardiac cells. As a result, the expression of RIP1, RIP3, MLKL mRNA and protein were decreased; by contrast, cell viability and the activity and expression of ALDH2 were increased, and oxidative stress was also inhibited. These results indicated that under hyperglycemic conditions, the inhibition of necroptosis via the activation of ALDH2 and downregulation of ROS production could mediate a cardiac-protective effect.

As ALDH2 participates in the protective effect of Nec-1, it is interesting to speculate on a potential association between ALDH2 and necroptosis. In a study regarding ALDH2 and 

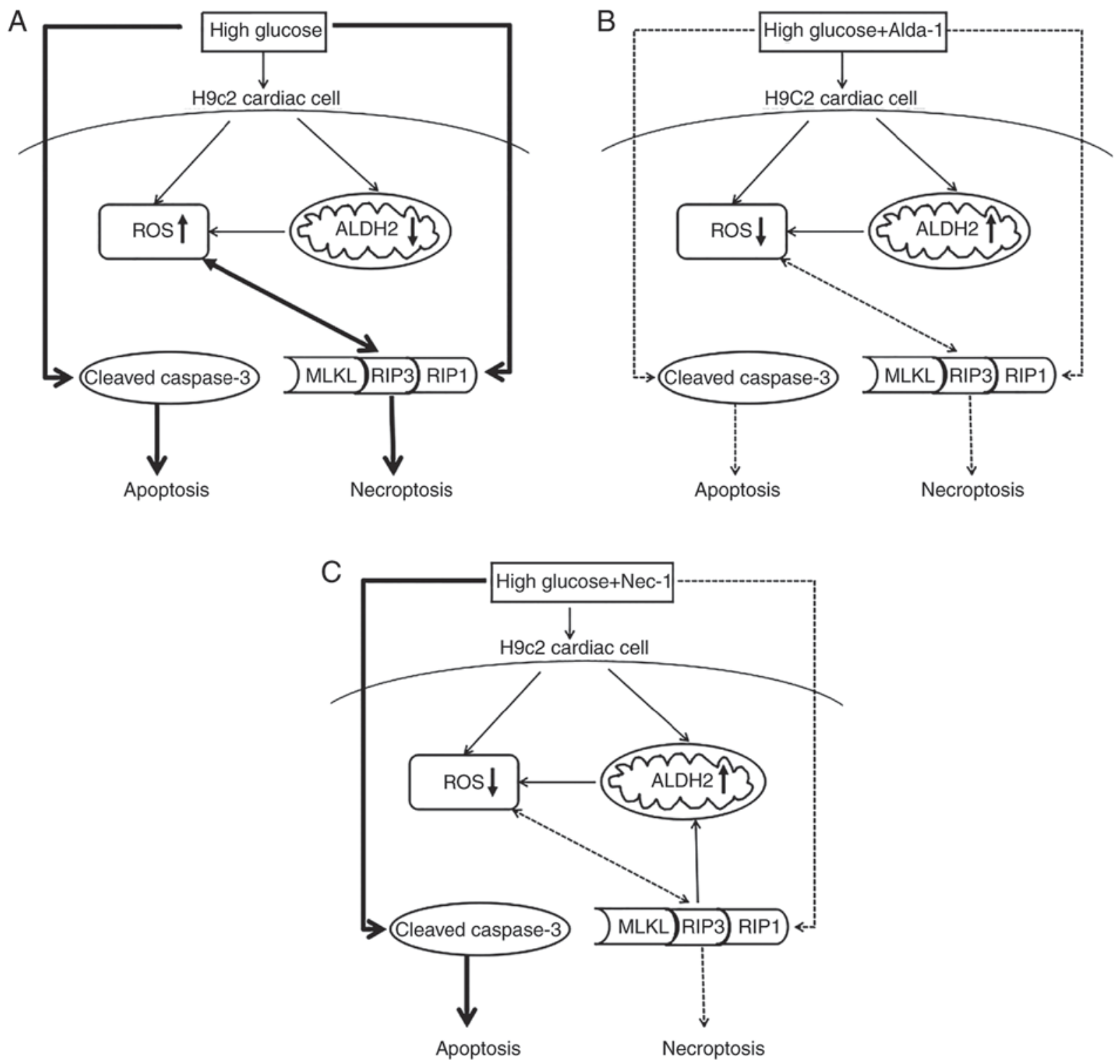

Figure 6. Possible mechanisms of the protective effects of activation of ALDH2 and inhibition of necroptosis in high glucose induced H9c2 cardiac cells injury. (A) Under high glucose conditions, ROS were over-released, the level of ALDH2 was downregulated and apoptosis, and necroptosis pathways were both activated. (B) With the presence of Alda-1 (the activator of ALDH2), accompanied by the increase of ALDH2, the overproduction of ROS was inhibited and apoptosis, and necroptosis pathways were inhibited. (C) When the necroptosis pathway was inhibited by Nec-1, the inhibitor of RIP1, accompanied by the inhibition of necroptosis, the overproduction of ROS was blocked, ALDH2 was increased but the apoptosis pathway was not affected. The solid black lines and arrowheads indicate activation; the dashed black lines and arrowheads indicate inhibition. MLKL, mixed lineage kinase domain like pseudokinase; RIP, receptor-interacting protein kinase 1; ROS, reactive oxygen species; ALDH2, aldehyde dehydrogenase 2; Nec-1, necrostatin-1.

necroptosis, Shen et al (26) reported that ALDH2 deficiency may lead to unexpected cardiac dysfunctions by enhancing myocardial apoptosis and necroptosis in a mouse model. In the present study, it was observed that ALDH2 activity and expression were increased when necroptosis was inhibited. On the basis of the previously reported studies $(7-9,26)$ and the present study, whether ALDH2 is a regulatory factor in necroptosis was further questioned. Therefore, a specific ALDH2 agonist, Alda-1 was selected to treat $\mathrm{H} 9 \mathrm{c} 2$ cardiac cells under HG conditions. The results indicated that with an increase in ALDH2 activity and expression, there was a decrease of the release of ROS, and the levels of RIP1, RIP3 and MLKL mRNA and protein expression were all decreased. This result suggested that inhibiting necroptosis was also involved in the protective mechanism of ALDH2 activation.
In the present study, another type of programmed cell death, apoptosis, was observed to be happening in $\mathrm{HG}$ induced cell injury. The results demonstrated Alda-1 but not Nec-1 could inhibit the protein expression of cleaved caspase-3, a terminal effector of apoptosis. The results were in accordance with the study by Liu et al (27), where they reported that Nec-1 did not alter the expression of caspase-3 following spinal cord injury in adult mice. However, there are different viewpoints. Chang et al (28) reported that in a mouse intracerebral hemorrhage model, Nec-1 not only suppressed necroptosis but also suppressed apoptosis and autophagy. Therefore, there may be cross-talk between necroptosis, apoptosis and autophagy following mouse intracerebral hemorrhage. Liu et al (29) indicated that the inhibition of RIP3 may serve as a therapeutic strategy for 
traumatic brain injury by suppressing inflammation, oxidative stress and apoptosis in a mouse model. Whether altering the levels of other proteins, including inhibiting RIP3 or MLKL, can exhibit an effect on apoptosis in HG-induced cardiac cell injury requires further investigation to analyze the connections of different cell death pathways.

In conclusion, necroptosis occurred in HG-induced H9c2 cardiac cell injury, suggesting that inhibiting necroptosis could have a cardiac-protective role. Furthermore, the activation of ALDH2 could improve cell injury by inhibiting necroptosis and apoptosis. The present study offers further insights into the role of ALDH2 in diseases. Inhibiting necroptosis may be a novel therapeutic tool for the prevention and treatment of diabetes-induced complications.

\section{Acknowledgements}

Not applicable.

\section{Funding}

The present study was supported by research grants from the Natural Science Foundation of China (grant no. 81770297), the Anhui Province University Top-Notch Talent Project (grant no. gxbjZD18), the Anhui Province Education Key Project (grant no. KJ2017A221) and the Bengbu Medical College Graduate Students Innovation Project (grant nos. Byycx1604 and Byycx1704).

\section{Availability of data and materials}

All data used and/or analyzed during this study are included in this published article.

\section{Authors' contributions}

$\mathrm{TF}, \mathrm{RC}$ and QG designed the study, performed the experiments including cell culture, RT-qPCR, western blotting and draft the manuscript. WW and HY performed the experiments including CCK8 assay, ALDH2 activity measurement and collected the experimental data. LS performed the DHE staining measurement, ZL and JH contributed to do the statistical analysis and interpret the experimental data. All authors revised the manuscript critically for important intellectual content and given the final approval of the version to be published. All authors read and approved the manuscript.

\section{Ethics approval and consent to participate}

All H9c2 cardiac cells experiments were approved by the Institutional Animal Care and Use Committee of Bengbu Medical College of China (Bengbu, China).

\section{Patient consent for publication}

Not applicable.

\section{Competing interests}

The authors declare that they have no competing interests.

\section{References}

1. Arora D, Sharma PK, Siddiqui MH and Shukla Y: Necroptosis: Modules and molecular switches with therapeutic implications. Biochimie 137: 35-45, 2017.

2. Weinlich R, Oberst A, Beere HM and Green DR: Necroptosis in development, inflammation and disease. Nat Rev Mol Cell Biol 18: 127-136, 2017.

3. Liang W, Chen M, Zheng D, He J, Song M, Mo L, Feng J and Lan J: A novel damage mechanism: Contribution of the interaction between necroptosis and ROS to high glucose-induced injury and inflammation in H9c2 cardiac cells. Int J Mol Med 40: 201-208, 2017.

4. Liang W, Chen M, Zheng D, Li J, Song M, Zhang W, Feng J and Lan J: The opening of ATP-sensitive $\mathrm{K}+$ channels protects $\mathrm{H} 9 \mathrm{c} 2$ cardiac cells against the high glucose-induced injury and inflammation by inhibiting the ROS-TLR4-necroptosis pathway. Cell Physiol Biochem 41: 1020-1034, 2017.

5. Luo XJ, Liu B, Ma QL and Peng J: Mitochondrial aldehyde dehydrogenase, a potential drug target for protection of heart and brain from ischemia/reperfusion injury. Curr Drug Targets 15: 948-955, 2014.

6. Matsumoto A, Thompson DC, Chen Y, Kitagawa K and Vasiliou V: Roles of defective ALDH2 polymorphism on liver protection and cancer development. Environ Health Prev Med 21: 395-402, 2016.

7. Gao Q, Wang HJ, Wang XM, Kang PF, Yu Y, Ye HW, Zhou H and Li ZH: Activation of ALDH2 with ethanol attenuates diabetes induced myocardial injury in rats. Food Chem Toxicol 56: 419-424, 2013.

8. Kang PF, Wu WJ, Tang Y, Xuan L, Guan SD, Tang B, Zhang H, Gao Q and Wang HJ: Activation of ALDH2 with low concentration of ethanol attenuates myocardial ischemia/reperfusion injury in diabetes rat model. Oxid Med Cell Longev 2016: 6190504,2016

9. Guo Y, Yu W, Sun D, Wang J, Li C, Zhang R, Babcock SA, Li Y, Liu M, Ma M, et al: A novel protective mechanism for mitochondrial aldehyde dehydrogenase (ALDH2) in type i diabetes-induced cardiac dysfunction: Role of AMPK-regulated autophagy. Biochim Biophys Acta 1852: 319-331, 2015

10. Livak KJ and Schmittgen TD: Analysis of relative gene expression data using real-time quantitative PCR and the 2(-Delta Delta C(T)) method. Methods 25: 402-408, 2001.

11. Fuchs Y and Steller H: Live to die another way: Modes of programmed cell death and the signals emanating from dying cells. Nat Rev Mol Cell Biol 16: 329-344, 2015.

12. Sun L, Wang H, Wang Z, He S, Chen S, Liao D, Wang L, Yan J, Liu W, Lei X and Wang X: Mixed lineage kinase domain-like protein mediates necrosis signaling downstream of RIP3 kinase. Cell 148: 213-227, 2012.

13. Wu W, Liu P and Li J: Necroptosis: An emerging form of programmed cell death. Crit Rev Oncol Hematol 82: 249-258, 2012.

14. Cano M, Wang L, Wan J, Barnett BP, Ebrahimi K, Qian J and Handa JT: Oxidative stress induces mitochondrial dysfunction and a protective unfolded protein response in RPE cells. Free Radic Biol Med 69: 1-14, 2014.

15. Osipov RM, Bianchi C, Feng J, Clements RT, Liu Y, Robich MP, Glazer HP, Sodha NR and Sellke FW: Effect of hypercholesterolemia on myocardial necrosis and apoptosis in the setting of ischemia-reperfusion. Circulation 120 (11 Suppl): S22-S30, 2009.

16. Zhang DW, Shao J, Lin J, Zhang N, Lu BJ, Lin SC, Dong MQ and Han J: RIP3, an energy metabolism regulator that switches TNF-induced cell death from apoptosis to necrosis. Science 325: 332-336, 2009.

17. LaRocca TJ, Sosunov SA, Shakerley NL, Ten VS and Ratner AJ: Hyperglycemic conditions prime cells for RIP1-dependent necroptosis. J Biol Chem 291: 13753-13761, 2016.

18. Wang $\mathrm{X}$, Yousefi $\mathrm{S}$ and Simon HU: Necroptosis and neutrophil-associated disorders. Cell Death Dis 9: 111, 2018.

19. Chen CH, Joshi AU and Mochly-Rosen D: The role of mitochondrial aldehyde dehydrogenase 2 (ALDH2) in neuropathology and neurodegeneration. Acta Neurol Taiwan 25: 111-123, 2016.

20. Lao-Sirieix P, Caldas C and Fitzgerald RC: Genetic predisposition to gastro-oesophageal cancer. Curr Opin Genet Dev 20: 210-217, 2010. 
21. Wang J, Wang H, Hao P, Xue L, Wei S, Zhang Y and Chen Y: Inhibition of aldehyde dehydrogenase 2 by oxidative stress is associated with cardiac dysfunction in diabetic rats. Mol Med 17: 172-179, 2011.

22. Degterev A, Huang Z, Boyce M, Li Y, Jagtap P, Mizushima N, Cuny GD, Mitchison TJ, Moskowitz MA and Yuan J: Chemical inhibitor of nonapoptotic cell death with therapeutic potential for ischemic brain injury. Nat Chem Biol 1: 112-119, 2005.

23. Adameova A, Hrdlicka J, Szobi A, Farkasova V, Kopaskova K, Murarikova M, Neckar J, Kolar F, Ravingerova $T$ and Dhalla NS: Evidence of necroptosis in hearts subjected to various forms of ischemic insults. Can J Physiol Pharmacol 95: 1163-1169, 2017.

24. Vieira M, Fernandes J, Carreto L, Anuncibay-Soto B, Santos M, Han J, Fernández-López A, Duarte CB, Carvalho AL and Santos AE: Ischemic insults induce necroptotic cell death in hippocampal neurons through the up-regulation of endogenous RIP3. Neurobiol Dis 68: 26-36, 2014.

25. Oerlemans MI, Koudstaal S, Chamuleau SA, de Kleijn DP, Doevendans PA and Sluijter JP: Targeting cell death in the reperfused heart: Pharmacological approaches for cardioprotection. Int J Cardiol 165: 410-422, 2013.

26. Shen C, Wang C, Han S, Wang Z, Dong Z, Zhao X, Wang P, Zhu H, Sun X, Ma X, et al: Aldehyde dehydrogenase 2 deficiency negates chronic low-to-moderate alcohol consumption-induced cardioprotecion possibly via ROS-dependent apoptosis and RIP1/RIP3/MLKL-mediated necroptosis. Biochim Biophys Acta 1863: 1912-1918, 2017.
27. Liu M, Wu W, Li H, Li S, Huang LT, Yang YQ, Sun Q, Wang CX Yu Z and Hang CH: Necroptosis, a novel type of programmed cell death, contributes to early neural cells damage after spinal cord injury in adult mice. J Spinal Cord Med 38: 745-753, 2015.

28. Chang P, Dong W, Zhang M, Wang Z, Wang Y, Wang T, Gao Y, Meng H, Luo B, Luo C, et al: Anti-necroptosis chemical necrostatin-1 can also suppress apoptotic and autophagic pathway to exert neuroprotective effect in mice intracerebral hemorrhage model. J Mol Neurosci 52: 242-249, 2014.

29. Liu ZM, Chen QX, Chen ZB, Tian DF, Li MC, Wang JM, Wang L, Liu BH, Zhang SQ, Li F, et al: RIP3 deficiency protects against traumatic brain injury (TBI) through suppressing oxidative stress, inflammation and apoptosis: Dependent on AMPK pathway. Biochem Biophys Res Commun 499: 112-119, 2018.

cc) (i) $\ominus$ This work is licensed under a Creative Commons Attribution-NonCommercial-NoDerivatives 4.0 International (CC BY-NC-ND 4.0) License. 